\title{
DNMT3a methylation in neuropathic pain
}

\author{
This article was published in the following Dove Press journal: \\ Journal of Pain Research \\ 18 September 2017 \\ Number of times this article has been viewed
}

\section{Cuijie Shao \\ Yong Gao \\ Dan Jin \\ Xin Xu \\ Shuying Tan \\ Hui Yu \\ Qingxiang Zhao \\ Li Zhao \\ Wansheng Wang \\ Deqiang Wang}

Department of Pain, Binzhou Medical University Hospital, Binzhou, China
Correspondence: Cuijie Shao; Deqiang Wang

Number 66I, Number 2, Huanghe Road, Binzhou Medical University Hospital, Binzhou, Shandong 256600, China Email shaocuijie@163.com; wdqbz@163.com
Background: $\mathrm{Mu}$ opioid receptor (MOR) plays a crucial role in mediating analgesic effects of opioids and is closely associated with the pathologies of neuropathic pain. Previous studies have reported that peripheral nerve injury downregulates MOR expression, but the epigenetic mechanisms remain unknown.

Objective: Therefore, we investigated DNA methyltransferase3a (DNMT3a) expression or methylation changes within MOR promoter in the spinal cord in a neuropathic pain induced by a chronic constriction injury (CCI) mouse model and further determined whether these injuryassociated changes are reversible by pharmacological interventions.

Methods: A CCI mouse model was established and tissue specimens of lumbar spinal cords were collected. The nociception threshold was evaluated by a Model Heated 400 Base. DNMT3a and MOR mRNA and protein level were detected by real-time-polymerase chain reaction and Western blot, respectively. Methylation of DNMT3a gene was measured by methylation-specific PCR.

Results: Our data showed that chronic nerve injury led to a significant upregulation of DNMT3a expression that was associated with increased methylation of MOR gene promoter and decreased MOR protein expression in the spinal cord. Inhibition of DNMT3a catalytic activity with DNMT inhibitor RG108 significantly blocked the increase in methylation of the MOR promoter, and then upregulated MOR expression and attenuated thermal hyperalgesia in neuropathic pain mice. Conclusion: This study demonstrates that an increase of DNMT3a expression and MOR methylation epigenetically play an important role in neuropathic pain. Targeting DNMT3a to the promoter of MOR gene by DNMT inhibitor may be a promising approach to the development of new neuropathic pain therapy.

Keywords: DNMT3A, pain, MOR, RG108, neuropathic pain

\section{Introduction}

Neuropathic pain, which is triggered by lesions to the somatosensory nervous system, is pathologically amplified responsiveness to noxious and innocuous stimuli. ${ }^{1}$ It has been a major health problem and causes not only considerable personal suffering but also enormous socioeconomic costs. ${ }^{2}$ In spite of the numerous analgesic drugs present in the market, including opioids, antidepressants, local anesthetics and others, the treatment outcome is highly variable, with a significant proportion of patients showing resistance to medication. ${ }^{3}$ In addition, many of these analgesic drugs are not disease-dependent and have serious side effects, such as neurotoxicity and addictive properties. New therapeutic strategies are desperately needed and the cellular and molecular mechanisms for pathological pain remain to be identified.

Opioid receptors, known as mu, delta, and kappa receptors, are major actors in pain control and are broadly distributed throughout the nervous system. ${ }^{4}$ The mu opioid 
receptor (MOR) plays a pivotal role in pain biology, as once it was shown that knocking out the MOR gene completely eliminates the analgesic action of morphine. ${ }^{5}$ MOR on presynaptic axon terminals in the spinal cord reduces transmitter release by inhibiting voltage-gated calcium channels, diminishing sensory input from nociceptors to the central nervous system. The receptor is also located postsynaptically, where it activates $G$ protein-coupled inwardly rectifying potassium channels to reduce excitability. ${ }^{6}$ Previous studies suggested that peripheral nerve injury downregulates MOR expression in afferent nerve cell bodies, ${ }^{7}$ and it is important to understand the potential mechanisms underlying these changes.

DNA methylation is a crucial epigenetic modification of the genome that is involved in regulating many cellular processes. ${ }^{8}$ Patterns of genomic DNA methylation are established and maintained by DNA methyltransferases (DNMTs) which could catalyze the covalent transfer of a methyl group from S-adenosyl-methionine to cytosine residues in generegulatory regions, thus leading to chromatin remodeling and gene silencing. ${ }^{9,10}$ DNA methyltransferase3a (DNMT3a) is mainly involved in the de novo establishment of DNA methylation marks, because it methylates hemimethylated DNA and also completely unmethylated DNA. ${ }^{11,12}$ Recent studies have shown that DNMT3a functions in synaptic plasticity, ${ }^{13}$ memory formation, ${ }^{14,15}$ and behavioral plasticity. ${ }^{16}$ Since pain processing and memory formation share a number of mechanisms and are both dependent on synaptic plasticity, it is likely that DNA methylation and therefore DNMT activity is regulated during the development of neuropathic pain states. ${ }^{9}$ Thus, we addressed the question of what are the potential epigenetic mechanisms that could lead to lasting downregulation of antinociceptive genes such as MOR?

In the present study, we examined DNMT3a and MOR expression in the spinal cord at different time points after chronic constriction injury (CCI). We also investigated the methylation status of MOR promoter in correlation with nociceptive behavior in a neuropathic pain mice model. Furthermore, we evaluated the effects of intrathecal administration of RG108, an isoform nonspecific DNMT inhibitor, on MOR methylation, DNMT3a and MOR expression, and neuropathic pain in CCI mice.

\section{Methods}

\section{Animals}

Male C57BL/6J mice (laboratory animal certificate number: SCXF 20130001) weighing 22-25 g were used, and randomly assigned to the different treatment groups. They were kept in transparent acrylic individual cages at $23^{\circ} \mathrm{C}$ with light-dark cycles of $12 \times 12$ hours. Water and food were available ad libitum until the mice were transported to the laboratory $\sim 1$ hour before experiments. All efforts were made to minimize animal suffering and to reduce the number of animals used. The experiments were conducted in accordance with the ethical guidelines of the International Association for the Study of Pain. ${ }^{17}$ All experimental protocols were approved by the Animal Care and Use Committee of Binzhou Medical College (Binzhou, Shandong Province, China; laboratory animals use license number: SYXK 20130019). Animals $(n=40)$ were randomly divided into four groups $(n=10)$. Any cause of death was excluded from the study $(n=4)$.

\section{Surgery}

$\mathrm{CCI}$ to the sciatic nerve was performed under pentobarbital $(50 \mathrm{mg} / \mathrm{kg})$ anesthesia, following the methods of Bennett and Xie. ${ }^{18}$ In brief, after exposing the left sciatic nerve from the surrounding tissue, three snug ligatures (4-0 chromic gut) were placed around the nerve. The interval among ligatures was about $1 \mathrm{~mm} \cdot{ }^{22}$ In the sham operation, the sciatic nerve was exposed as described above but no contact was made with the nerve. All animals showing motor weakness or signs of paralysis or sensory impairment during the observation period were abandoned.

\section{Pharmacological treatments}

RG108 was freshly dissolved in dimethyl sulfoxide at a stock concentration of $10 \mathrm{mM}$ according to previous research. ${ }^{8}$ Each drug-treated mouse was intrathecally injected with $5 \mu \mathrm{L}$ artificial cerebral spinal fluid containing $20 \mu \mathrm{M}$ RG108, which was the most effective dose that did not produce significant side effects on the mice' motor functions. Then, nerve injury was carried out with subsequent injections of RG108 or $0.9 \%$ saline from day 3 to day 7 after surgery. The pain thresholds, methylation levels, and protein expressions were assessed at day 7 post-injury.

\section{Behavioral test}

In thermal hyperalgesia tests, the nociception threshold was evaluated by the latency of paw withdrawal upon a thermal stimulus. The thermal withdrawal latency (TWL) of each mouse was measured before surgery, and on days 1, 3, 5, 7, and 14 after surgery. Unanesthetized animals were placed in Plexiglass cages on top of a glass sheet, and an adaptation period of 1 hour was allowed. A thermal stimulator (Model Heated 400 Base; IITC Life Science, Woodland Hills, CA, USA) was positioned under the glass sheet and the focus of the projection bulb was aimed exactly at the middle of the plantar surface of the 
animal. ${ }^{19}$ A cutoff time of 20 seconds was set to prevent tissue damage. The measurements were repeated three times with an interval of 5 minutes and the averages of responses were evaluated. All animals underwent baseline behavioral assessments immediately prior to surgery and no differences were observed between groups. All behavioral testing was performed by the same observer blinded to the animal treatments.

\section{Sample preparation}

After behavioral testing, the mice were decapitated under deep anesthesia, and their lumbar spinal cords were immediately removed. The tissues were frozen on dry ice and stored at $-80^{\circ} \mathrm{C}$ for use in methylation-specific PCR (MSP) or Western blot analysis. For immunohistochemistry, mice were deeply anesthetized with pentobarbital 7 days after surgery and quickly perfused with $20 \mathrm{~mL}$ normal saline followed by $20 \mathrm{~mL} \mathrm{4 \%} \mathrm{paraformaldehyde} \mathrm{through} \mathrm{the} \mathrm{ascending} \mathrm{aorta.}$ The mice were then killed, and their lumbar spinal cords were dissected out.

\section{MSP}

Genomic DNA was extracted from the lumbar spinal cord and $500 \mathrm{ng}$ of DNA was treated with sodium bisulfite following the manufacturer's instructions (Zymo Research, Orange, CA, USA). This modification resulted in a conversion of unmethylated cytosine to thymine, whereas the methylated cytosine remained unchanged. ${ }^{35}$ The resulting bisulfite-modified DNA was amplified by PCR. MSP primers were designed for the PCR amplification of MOR via Methyl Primer Express Software v1.0. MSP primers (for cytosine-phosphate-guanine $[\mathrm{CpG}]$ sites -344 and -255$)$ : forward 5'-AGGTTGATTTTGAGTTGTTTTGC-3' and reverse 5'-CAAAACGCCACTCTCTAAATAAA-AA-3'. PCR reactions were performed in an ABI 7500 fast real-time PCR system (Thermo Fisher Scientific, Waltham, MA, USA). Cycling conditions involved a predenaturation of 5 minutes at $95^{\circ} \mathrm{C} ; 40$ cycles of denaturation at $95^{\circ} \mathrm{C}$ for 20 seconds, annealing at $60^{\circ} \mathrm{C}$ for 30 seconds, and extension at $72^{\circ} \mathrm{C}$ for 20 seconds; followed by extension at $72^{\circ} \mathrm{C}$ for 10 minutes. In all cases, the validity of amplification was confirmed by the presence of a single peak in the melting temperature analysis and by linear amplification with increasing number of PCR cycles. ${ }^{45}$ Relative ratios of methylation levels were calculated by using the $\Delta \mathrm{Ct}$ method $\left(2^{-\Delta \Delta} \mathrm{Ct}\right)$ at a threshold of 0.02 .

\section{Western blot}

Proteins were extracted from the spinal cord using the nucleoprotein and cytoplasmic protein extraction kit (KeyGen
Biotech, Nanjing, China). The protein concentrations were quantified by bicinchoninic acid protein assay (Pierce; Thermo Fisher Scientific). The samples were diluted in lysis buffer and combined with sodium dodecyl sulfate (SDS) sample buffer. Nuclear or cytoplasmic extracts were then separated on an SDS-polyacrylamide gel electrophoresis system by means of a Bio-Rad Mini-Protean II cell device (Bio-Rad Laboratories Inc, Hercules, CA, USA) for 1 hour at 80 and $120 \mathrm{~V}$, respectively. The pre-stained marker was also loaded on the gel as molecular weight standard. The proteins were transferred onto polyvinylidene fluoride membranes, which were blocked by the addition of $5 \%$ nonfat dry milk in phosphate-buffered saline (PBS) with 5\% Tween 20 for 3 hours at room temperature. Membrane blots were incubated overnight at $4^{\circ} \mathrm{C}$ plus 1 hour at room temperature with rabbit anti-DNMT3a antibody(1:100; EpiGentek, Farmingdale, NY, USA), rabbit anti-mOR antibody (1:200; Abcam, Cambridge, UK), or mouse anti- $\beta$-actin antibody (1:20,000; SigmaAldrich, St Louis, MO, USA). After three 5-minute washes in PBS with $0.05 \%$ Tween 20, the membranes were further incubated with peroxidase-conjugated secondary antibodies donkey anti-rabbit (1:1,000; Santa Cruz Biotechnology, Dallas, TX, USA) or donkey anti-mouse (1:1,000; Santa Cruz) for 2 hours at room temperature. The blots were developed with SuperSignal West Pico Chemiluminescent Substrate according to the manufacturer's protocol (Pierce; Thermo Fisher Scientific). Blot images were digitally acquired by an LAS3000 Imager, and the results were normalized to loading control.

\section{Immunohistochemistry}

The lumbar spinal cord tissues were postfixed with 4\% paraformaldehyde for 12 hours, cryoprotected by immersion in $30 \%$ sucrose solution overnight at $4^{\circ} \mathrm{C}$, and sectioned on a freezing microtome set at $30 \mu \mathrm{m}$. Following an initial background block using 10\% normal donkey serum/PBS-Triton $\mathrm{X}-100$ at room temperature for 2 hours, the sections were then incubated with polyclonal primary rabbit anti-DNMT3a antibody (1:50; EpiGentek) in a humidity chamber for 24 hours at $4^{\circ} \mathrm{C}$. After several rinses in $1 \mathrm{M} \mathrm{PBS}$, the slides were subsequently reacted with secondary antibody, Alexa Fluor 594-conjugated anti-rabbit IgG (1:300; Thermo Fisher Scientific) for 2 hours at $37^{\circ} \mathrm{C}$. For double immunolabeling, we used the following antibodies: mouse monoclonal antibody against neuron-specific nuclear protein (anti-NeuN; 1:500; EMD Millipore, Billerica, MA, USA) and Alexa Fluor 488-conjugated anti-mouse IgG (1:300; Thermo Fisher Scientific). After washing, the sections were mounted with Shandon PermaFluor 
(Thermo Fisher Scientific) and analyzed using a confocal laser scanning microscope (Olympus Corporation, Tokyo, Japan). Specificity of staining was confirmed by omission of the primary antibody or the biotinylated secondary antibody in multiple sections. No positive staining was detected in any of these negative control experiments.

\section{Statistical analyses}

All the statistical analyses were conducted using SPSS 17.0 software (SPSS Inc, Chicago, IL, USA). The data were plotted as mean \pm SEM for pain behavior, methylation levels, and protein expression. Statistical analyses were performed by Student's $t$-test for comparing the difference between two groups and by analysis of variance followed by Bonferroni's test for multiple comparisons. Differences among experimental groups were considered to be statistically significant when $p<0.05$.

\section{Results}

\section{$\mathrm{CCl}$-evoked thermal hyperalgesia in mice}

To ensure that all CCI mice used in the study showed pain hypersensitivity, we measured the latency of paw withdrawal in response to thermal stimulus. Compared with presurgery baseline values, TWL of the ipsilateral, but not contralateral side was reduced on days 1, 3, 7, and 14 after CCI (Figure 1A). Respectively, these mice showed typical signs of hyperalgesia and allodynia such as toe closing, foot eversion, paw-clicking, and a marked limp, which were not observed in mice undergoing sham operation.

\section{Increased methylation levels of MOR gene promoter in neuropathic pain mice}

In order to test whether DNA methylation is involved in regulating MOR gene expression in neuropathic pain mice, the methylation status at the promoter of the MOR gene was determined by MSP assay. Previously, the existence of dual promoters was reported in mouse MOR gene, the proximal promoter (PP) and the distal promoter (DP). ${ }^{20}$ It has been shown that PP acts as the major promoter compared to the DP for MOR transcription; ${ }^{21}$ hence, we focused on the methylation statuses of the PP region in this study. There were 21 CpG sites in the $5^{\prime}$-flanking region $(-569$ to +31 , covering the PP region, with the ATG start codon designated +1 ) of the MOR gene (Figure 1B). ${ }^{22}$ The results indicate that the degree of methylation of two CpG sites ( -344 and -255$)$ within the PP region was markedly increased in the lumbar spinal cord at days 7 and 14 after CCI compared with controls (Figure 1C and 1D). We did not find the other $\mathrm{CpG}$ sites to have any significant methylation changes after nerve injury.

\section{Assessment of DNMT3a and MOR protein in the spinal cord after surgery}

We first examined the changes in DNMT3a protein expression in neuropathic pain mice. As shown in Figure 2A, a timedependent increase in DNMT3a expression was observed in the spinal cord from day 3 to day 14 after CCI compared to the control. In parallel, the expression of MOR protein was significantly decreased on days 7 and 14 following nerve ligation, while no MOR protein decrease was observed in sham-operated mice (Figure 2B). These results imply that the increasing DNMT3a expression may lead to the hypermethylation of MOR promoter, at least in part, contributing to the downregulation of MOR expression after peripheral nerve injury.

\section{Effects of RGI08 on pain hypersensitivity and MOR promoter methylation}

To verify that the overexpression of DNMT3a increased the methylation status in MOR gene in vivo, we treated the mice with an isoform nonspecific DNMT inhibitor (RG108) from day 3 to day 7 after surgery. We observed that thermal hyperalgesia was consistently attenuated in RG108-treated CCI mice from day 5 to day 7 after CCI surgery compared to the vehicle-treated controls (Figure 3A). However, there was no significant decrease in thermal sensitivity in the RG108-treated sham mice. To further test the involvement of MOR methylation in neuropathic pain, we determined the relationship between the state of methylation of the MOR promoter and thermal hypersensitivity. Our results (Figure 3B and $C$ ) show that upregulation of MOR promoter methylation by CCI was inhibited by RG108, which was significantly in accordance with the changes in pain behavior.

\section{Effects of RGI08 on DNMT3a and MOR protein levels in the spinal cord}

We next investigated whether nonspecific demethylation can reverse the reduced MOR protein expression correlated with thermal hyperalgesia following nerve injury. The data in Figure 4A demonstrated that the DNMT3a expression was almost completely suppressed in RG108-treated CCI and RG108treated sham mice compared to vehicle-treated groups, respectively. As shown in Figure 4B, intrathecal RG108 could block the decrease of MOR protein in neuropathic pain mice via inhibiting hypermethylation of the MOR promoter and promoting MOR gene transcription. Similarly, by immunohistochemical analysis (Figure 5A and B), DNMT3a expression in the nucleus in the dorsal horn of the lumbar spinal cord was also dramatically increased on day 7 post-CCI compared 

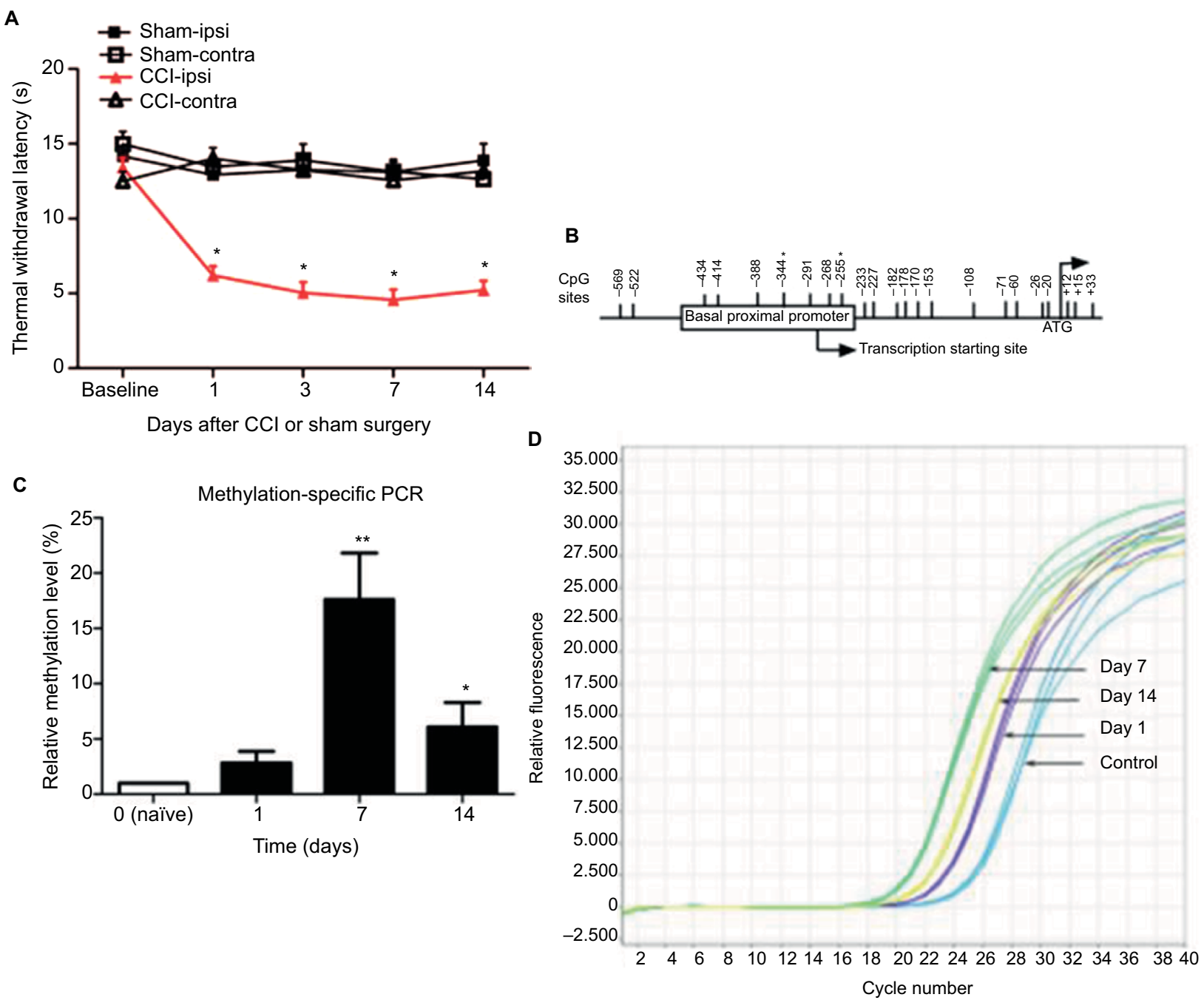

Figure I Levels measured over time of the decreased TWL and increased methylation of the MOR gene promoter in neuropathic pain mice.

Notes: (A) CCl induced temporal changes in thermal hyperalgesia. TWL of the ipsilateral (ipsi), but not contralateral (contra), side was reduced on days I, 3, 7, and I4 after $\mathrm{CCl}$ ( $n=6-14$ /group). (B) The 5 '-flanking region of the MOR gene contains 21 putative methyl CpG sites from -569 to +33 (with the ATG start codon designated +1 ). (C and D) Methylation statuses at the PP of the MOR gene were analyzed by MSP. Methylation levels of two sites ( -344 and -255 , located in the PP region) were markedly increased in the lumbar spinal cord at days 7 and 14 following $\mathrm{CCl}$ ( $n=6 /$ group). Data are presented as mean \pm SEM. One-way ANOVA followed by Bonferroni's test. * $p<0.05$ or $* * p<0.01$ versus before operation.

Abbreviations: MOR, mu opioid receptor; PCR, polymerase chain reaction; PP, proximal promoter; CCI, chronic constriction injury; ANOVA, analysis of variance; MSP, methylation-specific PCR; CPG, cytosine-phosphate-guanine; TWL, thermal withdrawal latency; SEM, standard error of the mean.

to that in naïve mice. The findings in immunostaining of DNMT3a were also consistent with the Western blot results (Figure 5C). These results support the hypothesis that DNA methylation is involved in neuropathic pain and the epigenetic changes correlated with thermal hyperalgesia could be reversed through DNA demethylation agents.

\section{Discussion}

Neuropathic pain is a particular type of pain that derives from structural or functional abnormalities of peripheral nerves or central nociceptive pathways. ${ }^{23}$ One feature of persistent pain states is dramatically altered gene expression in nociceptors, with at least $10 \%$ of the transcriptome being dysregulated in traumatic injury models of neuropathic pain. ${ }^{2}$ Regulation of gene expression can be achieved through DNA CpG methylation, a concept that is beginning to receive attention in pain research. Covalent modification of DNA by methylation is a critical epigenetic mechanism regulating gene expression; increased methylation inhibits and decreased methylation increases gene expression. ${ }^{24}$

CCI induced neuropathy, hyperalgesia, and allodynia, which could persist for a relatively long time..$^{25,26}$ In this study, MOR was significantly reduced in the lumbar spinal cord in CCI mice, which was in agreement with previous reports. ${ }^{7}$ The reduced expression of MOR in neuropathic conditions appears to contribute to the limited efficacy of opiates in these states. ${ }^{27}$ Promoter DNA hypermethylation is traditionally recognized to be repressive to gene expression. ${ }^{28,29}$ We therefore determined 
A

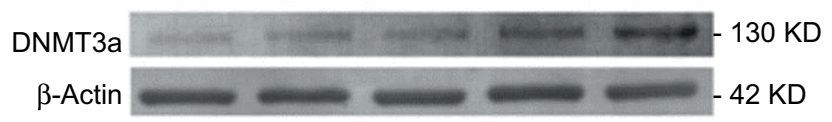

B

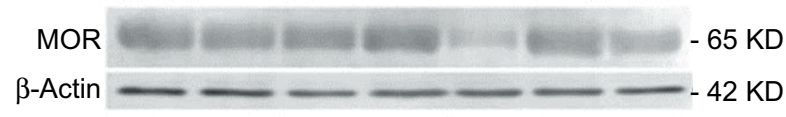

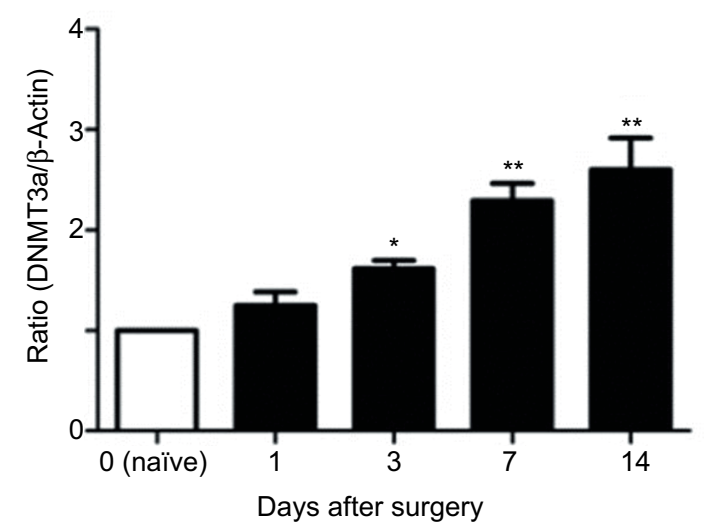

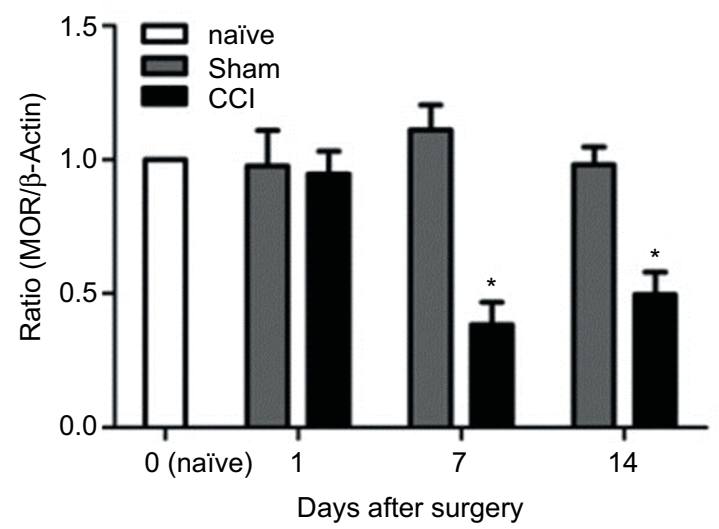

Figure 2 Levels measured over time of DNMT3a and MOR expression in the spinal cord in neuropathic pain mice.

Notes: Western blot shows that (A) nuclear DNMT3a expression was significantly increased in the spinal cord on days 3, 7, and I4 after CCl ( $\mathrm{n}=6 / \mathrm{group}$ ). (B) MOR protein was heavily reduced in the spinal cord on days 7 and 14 after nerve injury, while no MOR protein decrease was observed in sham-operated mice ( $\mathrm{n}=6 /$ group). $\beta$-Actin was used as an internal control. Data are presented as mean \pm SEM. One-way ANOVA followed by Bonferroni's test. $* p<0.05$ or $* * p<0.01$ versus before operation.

Abbreviations: MOR, mu opioid receptor; DNMT3a, DNA methyltransferase3a; CCl, chronic constriction injury; ANOVA, analysis of variance; SEM, standard error of the mean.

whether the MOR gene is commonly silenced by DNA methylation as the biological consequence following nerve ligation.

Generally, when DNA methylation is involved in gene silencing, cytosine methylation occurs in $\mathrm{CpG}$ dinucleotides within the $5^{\prime}$ regulatory regions of genes. ${ }^{30,31}$ There are 21 $\mathrm{CpG}$ sites in the PP region which is mainly responsible for transcription in mouse MOR gene. In order to detect potential methylated sites among them, we designed different MSP primers containing two or three CpG sites for the PCR amplification of MOR. Interestingly, our data demonstrate that two CpG sites ( -344 and -255$)$ within the MOR gene promoter were significantly hypermethylated in association with the decreased expression of MOR protein from day 7 to day 14 after CCI-induced neuropathic pain. The areas between these sites are one of the initiation sites where transcription factors bind to the DNA allowing transcription of the corresponding gene. When these regulatory regions are methylated, transcription factors are unable to bind DNA, thus inhibiting gene transcription. ${ }^{32}$ As gene transcription and protein translation are not regularly complemented in a short period, we did not find any obvious variations in either MOR gene methylation state or protein expression level on day 1 after nerve injury.

The results also showed that peripheral nerve lesions trigger changes in the dynamics of DNMT3a in the spinal cord. We found a time-dependent increased expression of DNMT3a that correlated with thermal hyperalgesia in CCI mice. DNA methylation patterns are closely regulated and changed by DNMTs which have potential functions in reprogramming efficiency. ${ }^{33}$ The increases in MOR gene DNA methylation and DNMT3a expression in CCI mice indicated an important role for both in neuropathic pain and might be involved in downregulating the expression of MOR protein.

However, it is now becoming clear that DNA methylation is, in fact, a reversible biological signal. ${ }^{34} \mathrm{RG} 108$ is the first discovered DNMT inhibitor through rational drug design and mainly used at the preclinical stage at present. ${ }^{35,36}$ It can obviously block the DNA methylation process even under lower substance concentration, via covalent binding with DNMT enzyme's active site and hindering its combination with DNA. ${ }^{37}$ In this study, the overexpression of DNMT3a and hypermethylation of MOR gene were significantly suppressed by intrathecal RG108 treatment from day 3 to day 7 in CCI mice. Furthermore, it partly reactivated MOR gene expression and alleviated neuropathic pain. Therefore, it is reasonable to conclude that the upregulation of DNMT3a expression may induce hypermethylation of MOR promoter, thus leading to the downregulation of MOR expression and pain hypersensitivity following peripheral nerve injury. The study provided evidence to support the hypothesis that DNA methylation occurs during neuropathic pain and that it is involved in silencing of antinociceptive genes such as MOR.

Recently, several studies have reported a relationship between DNA methylation and chronic pain including inflammatory pain, ${ }^{38-40}$ neuropathic pain, ${ }^{41,42}$ or other types of pain. ${ }^{43-45}$ Although this field of research is in its infancy, the research potentially discusses several pathophysiological processes underpinning abnormal pain processing and will guide prevention and intervention strategies in the future. However, 
A

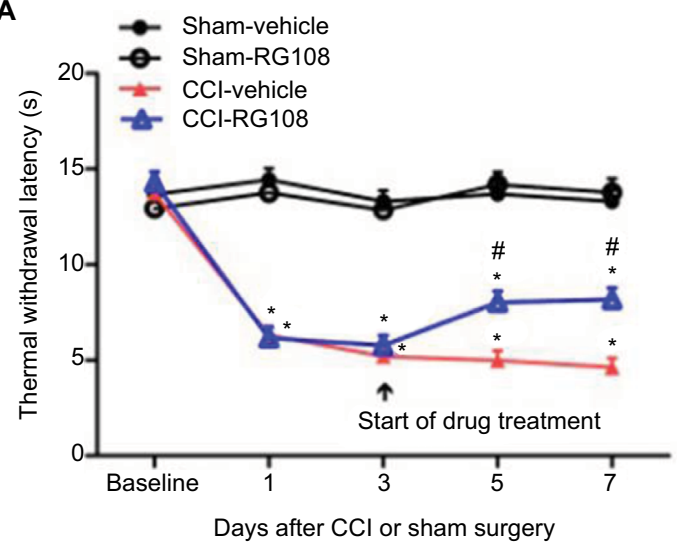

B

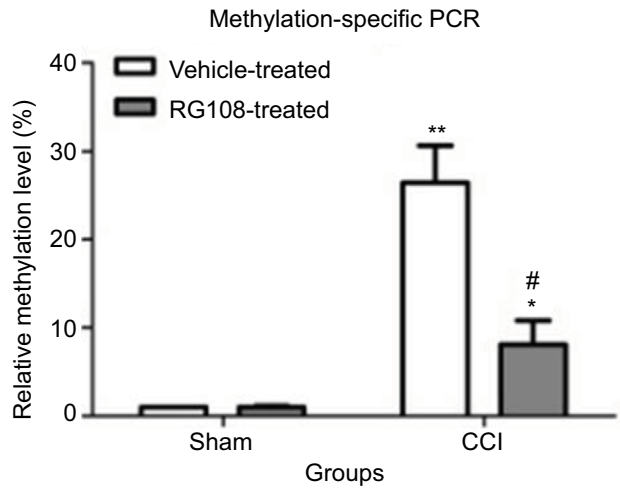

C

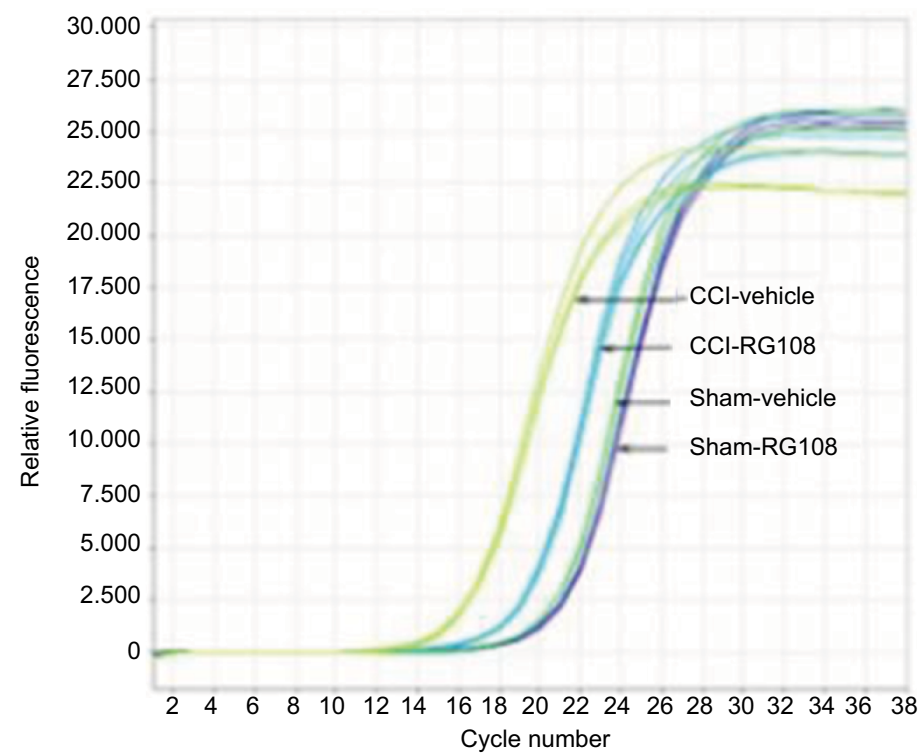

Figure 3 Intrathecal RG 108 treatment alleviates neuropathic pain and reverses methylation of the MOR promoter.

Notes: (A) Thermal hyperalgesia induced in CCl mice was consistently attenuated by intrathecal RGI08 treatment from day 5 to day 7 after surgery. Nevertheless, similar RG 108 treatment in sham-operated mice did not produce a significant change in thermal withdrawal latency during the observation period ( $\mathrm{n}=6 / \mathrm{group})$. (B and $\mathbf{C}$ ) Upregulation of methylation level of the MOR promoter by $\mathrm{CCl}$ surgery was inhibited by intrathecal $\mathrm{RGI} 108$ treatment at day 7 following $\mathrm{CCl}$ ( $\mathrm{n}=6 / \mathrm{group})$. Data are presented as mean \pm SEM. One-way ANOVA followed by Bonferroni's test. ${ }^{*} p<0.05$ or $*^{*} p<0.01$ versus before operation; ${ }^{*} p<0.05$ compared to the corresponding vehicle-treated group.

Abbreviations: MOR, mu opioid receptor; PCR, polymerase chain reaction; CCl, chronic constriction injury; ANOVA, analysis of variance; SEM, standard error of the mean.

few of the studies provided evidence that DNA methylation of a single gene plays a role in neuropathic pain. Our study suggests the underlying epigenetic mechanism for MOR protein downregulation following nerve injury, which was unlikely in accordance with the previous findings in inflammatory pain models. ${ }^{9,35}$ There are multiple mechanisms by which peripheral nerve injury can result in reducing MOR expression and the DNA methylation mechanism may not be the uppermost major contributing factor. The MOR gene is likely to be just one example of many pronociceptive or antinociceptive genes that are similarly regulated by DNA methylation in neuropathic pain. Given the restrictions on the MSP method, we could not detect the changes of all the $\mathrm{CpG}$ sites in the PP region. In addition, RG108 inhibits all DNMTs, not a specific DNMT, even though it almost completely blocked the aberrant increase of DNMT3a. Therefore, further studies are warranted to inves- tigate the other potential sites specifically targeted for MOR gene expression and explore other DNMT subtypes involved in targeting gene transcription in persistent pain state.

\section{Conclusion}

The study has provided new and fundamental insights into the roles that DNA methylation and DNMT3a have in the establishment and development of neuropathic pain. To our knowledge, this is the first report that demonstrates epigenetic regulation of MOR promoter region in neuropathic condition. DNA methylation of the MOR promoter is increased with overexpression of DNMT3a after nerve injury, resulting in the silencing of MOR gene in afferent nerve cell bodies. Additionally, the epigenetic prospective also provides novel avenues to treat pathological pain through reversing aberrant methylation via pharmacological means. 
A
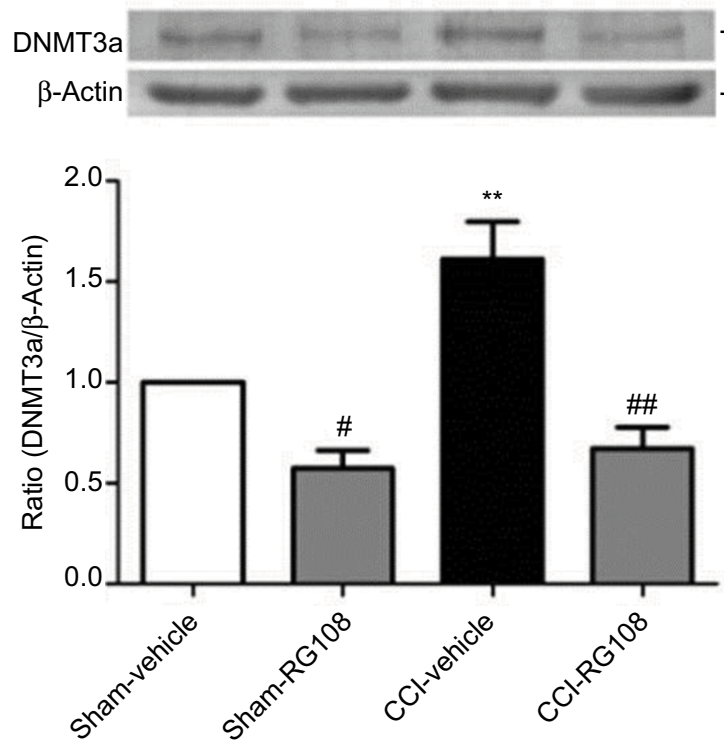

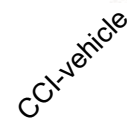

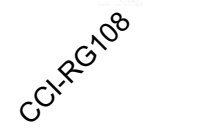

B
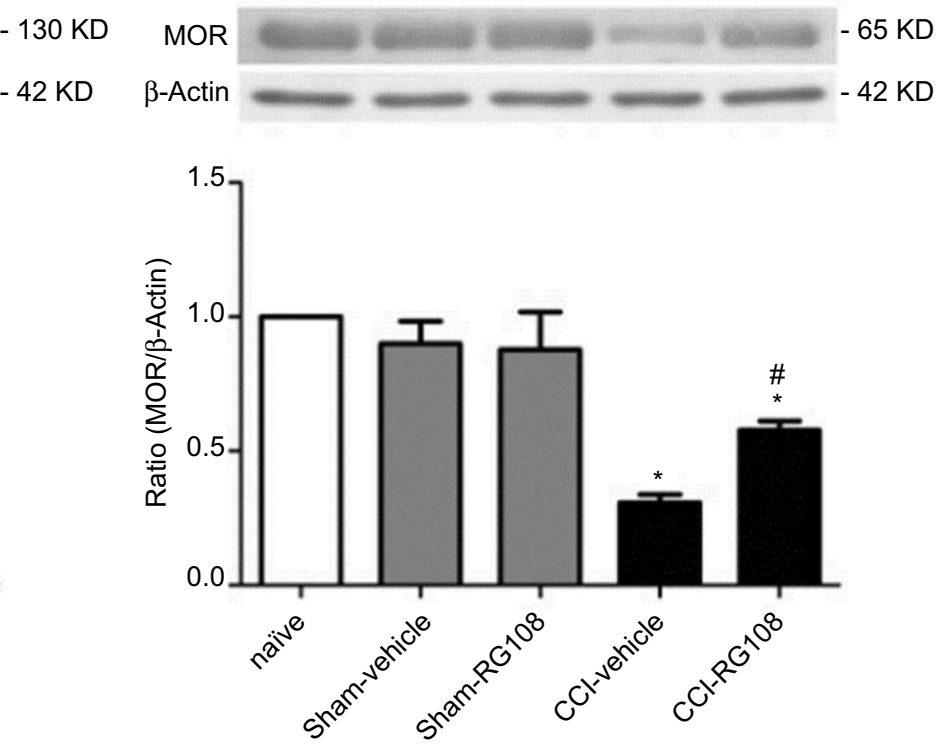

Figure 4 Effects of RGI08 on the expression of DNMT3a and MOR in the spinal cord.

Notes: (A) Western blot shows that intrathecal RGI08 treatment inhibits DNMT3a expression at the spinal level in both sham-operated and nerve-injured mice at day 7 after surgery ( $\mathrm{n}=6$ /group). (B) MOR protein in the spinal cord was upregulated by intrathecal RGI08 treatment at day 7 following CCl, while no MOR protein increase was observed in RG 108-treated sham mice ( $n=6 /$ group). $\beta$-Actin was used as an internal control. Data are presented as mean \pm SEM. One-way ANOVA followed by Bonferroni's test. ${ }^{*} p<0.05$ or $* * p<0.0$ I versus before operation; ${ }^{*} p<0.05$ or ${ }^{\# \#} p<0.01$ compared to the corresponding vehicle-treated group.

Abbreviations: MOR, mu opioid receptor; DNMT3a, DNA methyltransferase3a; CCI, chronic constriction injury; ANOVA, analysis of variance; SEM, standard error of the mean.
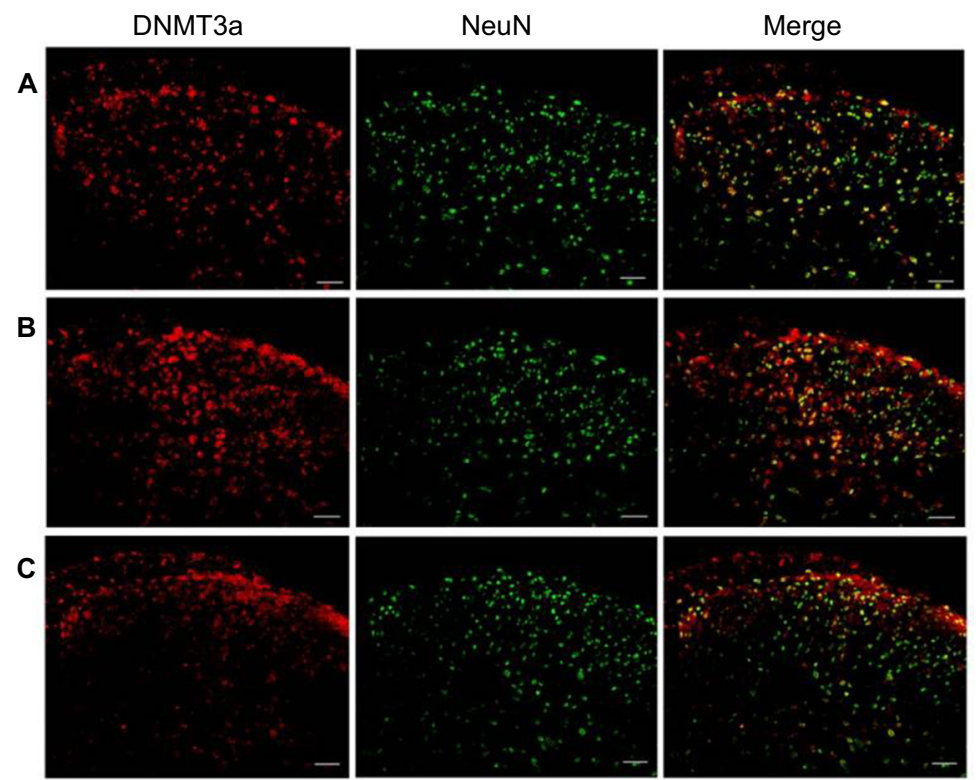

Figure 5 Immunohistochemical double labeling between DNMT3a (red) and NeuN (green) in the spinal cord in mice 7 days after surgery.

Notes: Immunopositive staining was observed in the dorsal horn from sham-operated mice (A), vehicle-treated $\mathrm{CCl}$ mice (B), and RGI08-treated CCl mice (C). CCl surgery dramatically increased DNMT3a-positive signals in NeuN-positive neurons in spinal cord dorsal horn at day 7 after injury. Moreover, the highly increased DNMT3a expression could be downregulated by RGI08 treatment. Scale bars $=50 \mu \mathrm{m}$.

Abbreviations: DNMT3a, DNA methyltransferase3a; $\mathrm{CCl}$, chronic constriction injury.

\section{Acknowledgments}

This work was supported by the Youth Fund of National Natural Science Foundation of China (grant no. 81100813 to Cuijie Shao), Natural Science Foundation for Colleges and Universities of Jiangsu Education Department (grant no. 11KJB320018 to Cuijie Shao), China Postdoctoral Science Foundation (grant no. 2012M510189 to Cuijie Shao), a Fund of the Qing Lan Project of Jiangsu, Sci- 
ence and Technology Planning Project of Shandong Educational Commission (grant no. J14LL02 to Cuijie Shao), Natural Science Foundation of Shandong Province. (No. ZR2017MH125 to Cuijie Shao), Youth Fund of Affiliated Hospital of Binzhou Medical College (grant no. 2013QNKYJJ09), and the Doctor Startup Fund Program funded by the Affiliated Hospital of Binzhou Medical College. The authors are thankful for the grants, and to Xiao Song for raising the animals, and to Junli Cao, Licai Zhang, and Dan Wang for guiding the experiments.

\section{Author contributions}

Cuijie Shao conceived, designed, and performed the experiments. Deqiang Wang guided the experiments. All authors contributed toward data analysis, drafting and critically revising the paper, gave final approval of the version to be published, and agree to be accountable for all aspects of the work.

\section{Disclosure}

The authors report no conflicts of interest in this work.

\section{References}

1. Costigan M, Scholz J, Woolf CJ. Neuropathic pain: a maladaptive response of the nervous system to damage. Annu Rev Neurosci. 2009; 32:1-32.

2. Denk F, McMahon SB. Chronic pain: emerging evidence for the involvement of epigenetics. Neuron. 2012;73:435-444.

3. Chiechio S, Copani A, Zammataro M, Battaglia G, Gereau RW, Nicoletti F. Transcriptional regulation of type-2 metabotropic glutamate receptors: an epigenetic path to novel treatments for chronic pain. Trends Pharmacol Sci. 2010;31:153-160.

4. Gaveriaux-Ruff C, Nozaki C, Nadal X, et al. Genetic ablation of delta opioid receptors in nociceptive sensory neurons increases chronic pain and abolishes opioid analgesia. Pain. 2011;152:1238-1248.

5. Kieffer BL, Evans CJ. Opioid tolerance - in search of the holy grail. Cell. 2002;108:587-590.

6. WoolfCJ. Mu and delta opioid receptors diverge. Cell. 2009;137:987-988.

7. Porreca F, Tang QB, Bian D, Riedl M, Elde R, Lai J. Spinal opioid mu receptor expression in lumbar spinal cord of rats following nerve injury. Brain Res. 1998;795:197-203.

8. Robertson KD. DNA methylation and human disease. Nat Rev Genet. 2005;6:597-610.

9. Tochiki KK, Cunningham J, Hunt SP, Geranton SM. The expression of spinal methyl-CpG-binding protein 2, DNA methyltransferases and histone deacetylases is modulated in persistent pain states. Mol Pain. 2012;8:14.

10. Chestnut BA, Chang Q, Price A, Lesuisse C, Wong M, Martin LJ. Epigenetic regulation of motor neuron cell death through DNA methylation. J Neurosci. 2011;31:16619-166136.

11. Jurkowska RZ, Jurkowski TP, Jeltsch A. Structure and function of mammalian DNA methyltransferases. Chembiochem. 2011;12:206-222.

12. Chedin F. The DNMT3 family of mammalian de novo DNA methyltransferases. Prog Mol Biol Transl Sci. 2011;101:255-285.

13. Levenson JM, Roth TL, Lubin FD, et al. Evidence that DNA (cytosine-5) methyltransferase regulates synaptic plasticity in the hippocampus. J Biol Chem. 2006;281:15763-15773.

14. Miller CA, Sweatt JD. Covalent modification of DNA regulates memory formation. Neuron. 2007;53:857-869.
15. Day JJ, Sweatt JD. DNA methylation and memory formation. Nat Neurosci. 2010;13:1319-1323.

16. LaPlant Q, Vialou V, Covington HE 3rd, et al. Dnmt3a regulates emotional behavior and spine plasticity in the nucleus accumbens. Nat Neurosci. 2010;13:1137-1143.

17. Zimmermann M. Ethical guidelines for investigations of experimental pain in conscious animals. Pain. 1983;16:109-110.

18. Bennett GJ, Xie YK. A peripheral mononeuropathy in rat that produces disorders of pain sensation like those seen in man. Pain. 1988;33:87-107.

19. Uchida H, Ma L, Ueda H. Epigenetic gene silencing underlies C-fiber dysfunctions in neuropathic pain. J Neurosci. 2010;30:4806-4814.

20. Ko JL, Liu HC, Minnerath SR, Loh HH. Transcriptional regulation of mouse mu-opioid receptor gene. J Biol Chem. 1998;273:27678-27685.

21. Ko JL, Chen HC, Loh HH. Differential promoter usage of mouse muopioid receptor gene during development. Brain Res Mol Brain Res. 2002;104:184-193.

22. Hwang CK, Song KY, Kim CS, et al. Evidence of endogenous mu opioid receptor regulation by epigenetic control of the promoters. $\mathrm{Mol}$ Cell Biol. 2007;27:4720-4736.

23. Campbell JN, Meyer RA. Mechanisms of neuropathic pain. Neuron. 2006;52:77-92.

24. Stone LS, Szyf M. The emerging field of pain epigenetics. Pain. 2013;154: $1-2$.

25. Zhu XY, Huang CS, Li Q, et al. p300 exerts an epigenetic role in chronic neuropathic pain through its acetyltransferase activity in rats following chronic constriction injury (CCI). Mol Pain. 2012;8:84.

26. Zhang YQ, Guo N, Peng G, et al. Role of SIP30 in the development and maintenance of peripheral nerve injury-induced neuropathic pain. Pain. 2009;146:130-140.

27. Lee CY, Perez FM, Wang W, et al. Dynamic temporal and spatial regulation of mu opioid receptor expression in primary afferent neurons following spinal nerve injury. Eur J Pain. 2011;15:669-675.

28. Lugthart S, Figueroa ME, Bindels E, et al. Aberrant DNA hypermethylation signature in acute myeloid leukemia directed by EVI1. Blood. 2011;117:234-241.

29. Nandakumar V, Vaid M, Tollefsbol TO, Katiyar SK. Aberrant DNA hypermethylation patterns lead to transcriptional silencing of tumor suppressor genes in UVB-exposed skin and UVB-induced skin tumors of mice. Carcinogenesis. 2011;32:597-604.

30. Bird A. DNA methylation patterns and epigenetic memory. Genes Dev. 2002;16:6-21.

31. Brenner C, Fuks F. DNA methyltransferases: facts, clues, mysteries. Curr Top Microbiol Immunol. 2006;301:45-66.

32. Kimura H, Shiota K. Methyl-CpG-binding protein, MeCP2, is a target molecule for maintenance DNA methyltransferase, Dnmt1. J Biol Chem. 2003;278:4806-4812.

33. Yamanaka K, Balboula AZ, Sakatani M, Takahashi M. Gene silencing of DNA methyltransferases by RNA interference in bovine fibroblast cells. J Reprod Dev. 2010;56:60-67.

34. Ramchandani S, Bhattacharya SK, Cervoni N, Szyf M. DNA methylation is a reversible biological signal. Proc Natl Acad Sci USA. 1999;96: 6107-6112.

35. Lyko F, Brown R. DNA methyltransferase inhibitors and the development of epigenetic cancer therapies. J Natl Cancer Inst. 2005;97:1498-1506.

36. Siedlecki P, Garcia Boy R, Musch T, et al. Discovery of two novel, small-molecule inhibitors of DNA methylation. J Med Chem. 2006;49:678-683.

37. Brueckner B, Garcia Boy R, Siedlecki P, et al. Epigenetic reactivation of tumor suppressor genes by a novel small-molecule inhibitor of human DNA methyltransferases. Cancer Res. 2005;65:6305-6311.

38. Qi F, Zhou Y, Xiao Y, et al. Promoter demethylation of cystathioninebeta-synthetase gene contributes to inflammatory pain in rats. Pain. 2013;154:34-45.

39. Imagawa K, de Andres MC, Hashimoto K, et al. The epigenetic effect of glucosamine and a nuclear factor-kappa $\mathrm{B}(\mathrm{NF}-\mathrm{kB})$ inhibitor on primary human chondrocytes - implications for osteoarthritis. Biochem Biophys Res Commun. 2011;405:362-367. 
40. Hashimoto K, Oreffo RO, Gibson MB, Goldring MB, Roach HI. DNA demethylation at specific $\mathrm{CpG}$ sites in the IL1B promoter in response to inflammatory cytokines in human articular chondrocytes. Arthritis Rheum. 2009;60:3303-3313.

41. Wang Y, Liu C, Guo QL, et al. Intrathecal 5-azacytidine inhibits global DNA methylation and methyl-CpG-binding protein 2 expression and alleviates neuropathic pain in rats following chronic constriction injury. Brain Res. 2011;1418:64-69.

42. Tajerian M, Alvarado S, Millecamps M, et al. Peripheral nerve injury is associated with chronic, reversible changes in global DNA methylation in the mouse prefrontal cortex. PloS One. 2013;8:e55259.
43. Tajerian M, Alvarado S, Millecamps M, et al. DNA methylation of SPARC and chronic low back pain. Mol Pain. 2011;7:65.

44. Viet CT, Ye Y, Dang D, et al. Re-expression of the methylated EDNRB gene in oral squamous cell carcinoma attenuates cancer-induced pain. Pain. 2011;152:2323-2332.

45. Doehring A, Oertel BG, Sittl R, Lotsch J. Chronic opioid use is associated with increased DNA methylation correlating with increased clinical pain. Pain. 2013;154:15-23.

\section{Publish your work in this journal}

The Journal of Pain Research is an international, peer reviewed, open access, online journal that welcomes laboratory and clinical findings in the fields of pain research and the prevention and management of pain. Original research, reviews, symposium reports, hypothesis formation and commentaries are all considered for publication.
Dovepress

The manuscript management system is completely online and includes a very quick and fair peer-review system, which is all easy to use. Visit http://www.dovepress.com/testimonials.php to read real quotes from published authors. 